\title{
Manejo de la vía aérea en una espondilitis anquilosante: Caso clínico
}

\section{Case report: ankylosing spondylitis and difficult airway}

Claudia Robin'1, Miguel Fica ${ }^{1}$

\begin{abstract}
Handling a difficult airway is one of the biggest challenges for an anesthesiologist due to morbidity and mortality that it involves. This is why available devices are permanently reviewed to validate them in the handling of a known difficult airway or previously unknown. Patients with severe ankylosing spondylitis, almost everyone of them, they have a difficult airway due to a stiff neck spine and/ or limitation in the mouth opening. In this case report we present a patient with severe ankylosing spondylitis, admitted for a total hip replacement surgery. We followed recommendations for difficult airway management using the devices available in the Anesthesiology Unit of our Hospital. Previous patient consent, we performed an awake intubation using a video laryngoscope and gum elastic bougie, under sedation, succesfully and with no complications.
\end{abstract}

\section{RESUMEN}

Manejar una vía aérea difícil es uno de los mayores desafíos para un anestesiólogo debido a la morbilidad y mortalidad que conlleva. Esta es la razón por la cual los dispositivos disponibles se revisan permanentemente para validarlos en el manejo de una vía aérea difícil conocida o desconocida previamente. Los pacientes con espondilitis anquilosante severa, casi todos ellos, tienen una vía aérea difícil debido a una rigidez en la columna cervical y/o limitación en la apertura de la boca. En este caso, presentamos a un paciente con EA grave, admitido para una cirugía de reemplazo total de cadera. Seguimos las recomendaciones para el manejo de la vía aérea difícil usando los dispositivos disponibles en la Unidad de Anestesiología de nuestro Hospital. Con el consentimiento previo del paciente, realizamos una intubación con un laringoscopio de video y goma elástica bougie, bajo sedación, con éxito y sin complicaciones.

\section{Key words:}

Difficult airway, ankylosing spondylitis, videolaringoscope

\section{Palabras clave:}

Vía aérea difícil, espondilitis anquilosante, videolaringoscopio

Hospital de Castro, Chile.

Fecha de recepción: 12 de agosto de 2018

Fecha de aceptación: 23 de noviembre de 2018

\section{ORCID}

https://orcid.org/0000-0002-8185-8169

\section{Correspondencia:}

Claudia Robin

Email: claudia.robin@gmail.com 


\section{Introducción}

a dificultad en el manejo de la vía aérea es una de las mayores causas de morbimortalidad anestésica[1]-[12]. Las guías de manejo de la vía aérea difícil de la ASA 2013 tienen como novedad considerar como componente de la VAD la dificultad de colocar un dispositivo supraglótico y la dificultad de la laringoscopía, así también incluye el fastrack como alternativa y el videolaringoscopio como dispositivo de inicio al sospechar de VAD[2]. La Sociedad Chilena de Anestesiología ya en el 2012 en su "algoritmo de manejo vía aérea difícil" propone como alternativa al uso del fibrobroncoscopio en el enfrentamiento del paciente con predictores de mayor complejidad de VAD la evaluación vigil con videolaringoscopio[3].

Nosotros reportaremos nuestra experiencia con un paciente con antecedentes de espondilitis anquilosante avanzada y por lo tanto una vía aérea difícil anticipada, que abordamos con el device que contábamos en nuestro servicio para estos casos; un videolaringoscopio (glidescope) con paciente despierto bajo sedación.

\section{Método}

Presentamos el caso clínico de manejo de vía aérea difícil en un paciente de 57 años afectado de espondilitis anquilosante programado para ser intervenido de prótesis total de cadera derecha.

En el examen preoperatorio, paciente con antecedentes de HTA en tratamiento con buen control, el electrocardiograma normal, exámenes de sangre, coagulación y orina dentro de rangos normales. Se solicitaron radiografías de columna lumbar, torácica y

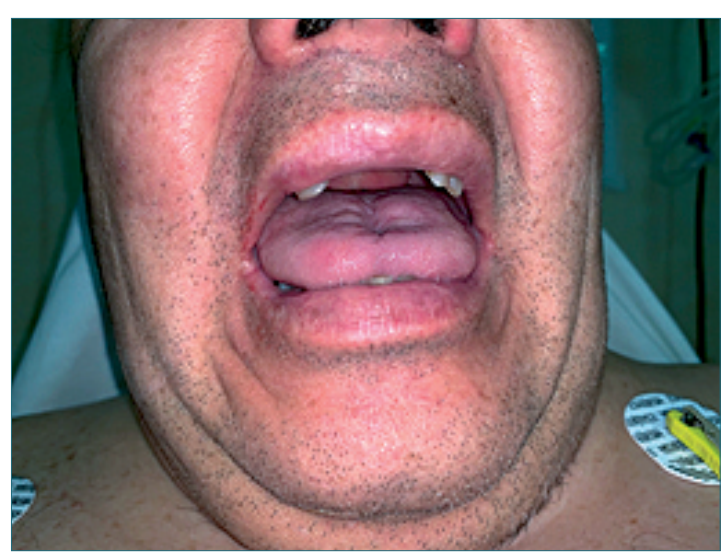

Figura 1. Evaluación de Mallampati grado 3 del paciente. cervical en las que se evidenciaron anquilosis de toda la columna vertebral (se adjuntan imágenes de columna cervical). La valoración de la vía aérea mostraba: sin limitación apertura bucal, grado MallampatiSamsoon III (Figura 1), distancia tiromentoniana $>6,5$ $\mathrm{cm}$, movilidad nula de la articulación atlantooccipital e imposibilidad de flexoextensión cervical. No presentaba historia de apnea del sueño, retrognatia, barba ni dentadura extraíble. Se descarta plantear anestesia regional por las complicaciones técnicas evidenciadas en la radiografía y ante la presencia de una potencial vía aérea difícil (VAD) y considerando los device de vía aérea difícil con que contábamos se decide intentar intubación vigil con un videolaringoscopio al paciente bajo sedación y anestesia local. Se informa a paciente y obtiene consentimiento informado del paciente.

Intubación se realiza bajo sedación con remifentanil en BIC y colutorios de lidocaína, atropina para disminuir las secreciones, como se muestra en la fotografía el paciente debe ser inducido sentado por la rigidez cervical, se preoxigena, se introduce el laringoscopio por vía anterior y visualizada las cuerdas vocales, se insinúa un GEB entre ellas, se induce la anestesia, se introduce el GEB, luego el TET y se conecta a ventilación mecánica sin incidentes con paciente estable hemodinámicamente durante todo el procedimiento; la cirugía y anestesia procede sin incidentes y novedades, la VM no tuvo dificultades especiales, se usó técnica protectora con bajo VC, Peep y FR según EtCO2, se extuba con prueba de filtración con cuff desinflado, más intercambiador puesto, se revirtió el relajante (rocuronio) con sugamadex, resultando el procedimiento tranquilo y exitoso.

\section{Discusión}

La espondilitis anquilosante (EA) es un proceso inflamatorio[4] que afecta principalmente al esqueleto axial, pero que también puede atacar a las articulaciones periféricas (más frecuentemente artritis de cadera y hombro) y estructuras extraarticulares[5]. El hallazgo más característico en la afectación del esqueleto axial es la entesitis inicialmente en el cuerpo vertebral, siguiendo con osteítis en el margen vertebral y osificación del anillo fibroso con la formación de puentes óseos intervertebrales (sindesmofitos) que originan la imagen radiológica típica de la columna en "caña de bambú" [6] que comienza en las articulaciones sacoilicas ascendiendo hasta la columna cervical, por ello frecuentemente tienen dificultad o imposibilidad de intubar debido a la limitación en la apertura bucal y/o la rigidez de la columna cervical disminuyendo su 

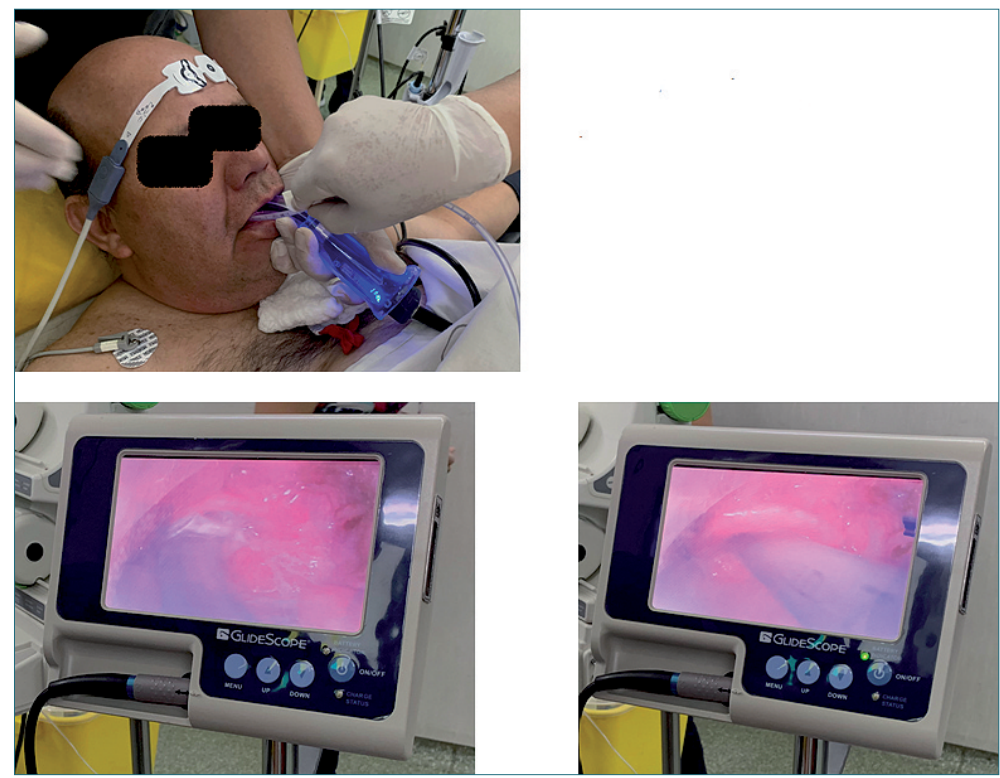

Figura 2. Preoxigenación y laringoscopía con pacientes sedado y vigil. Secuencia de videolaringoscopía.

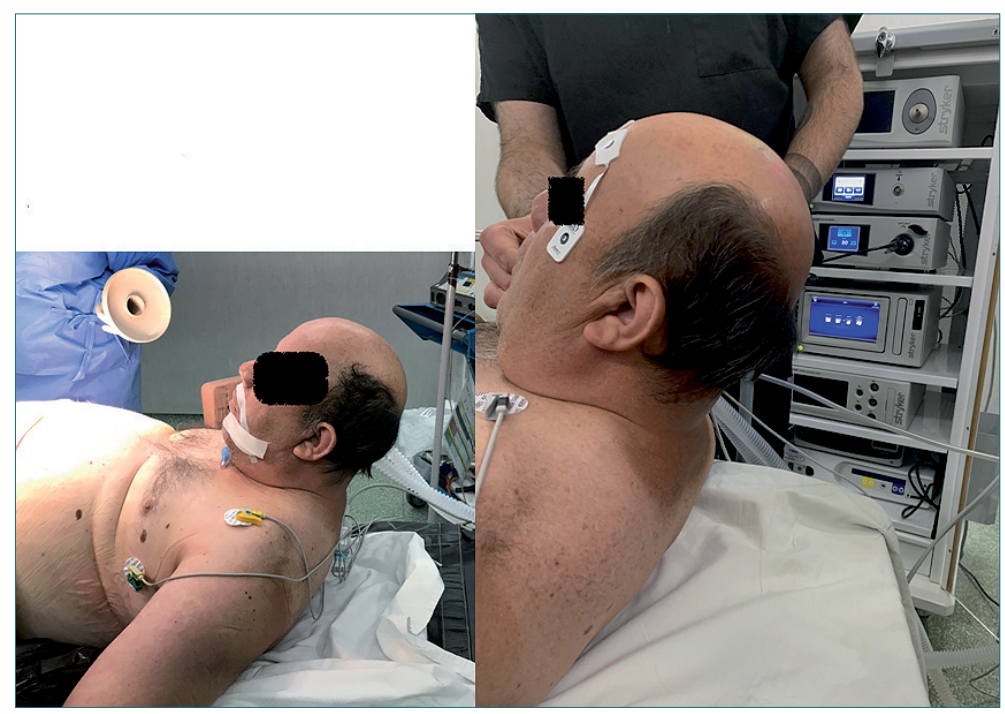

Figura 3. Paciente anestesiado, con relajo muscular, en VM... muestra la rígida postura producto de la $\mathrm{EA}$.

movilidad y en casos severos provocando una fijación total. La afectación de la articulación cricoaritenoidea puede no ser evidenciada en las escalas convencionales de valoración de la vía aérea y dificulta aún más el acceso de la misma. Con la progresión de la EA aparecen también alteraciones de articulaciones atlantoccipital (limitación de la movilidad cervical) y temporomandibular (limitación de la apertura bucal), exageración de la cifosis torácica, riesgo de fractura del raquis cervical (osteoporosis difusa)[7],[8] y anqui- losis condrocostal responsable de una neumopatía restrictiva (menor tolerancia a la apnea)[9] que complican el manejo de la vía aérea (Figuras 2, 3 y 4).

En cuanto a las recomendaciones de manejo de la VA en los pacientes con EA, se recomienda la valoración previa con $\mathrm{Rx}$, no se recomienda la extensión del cuello por el riesgo de lesiones nerviosas e insuficiencia vertebrobasilar, también se advierte que la traqueostomía podría ser imposible si hay una deformidad[10]. 


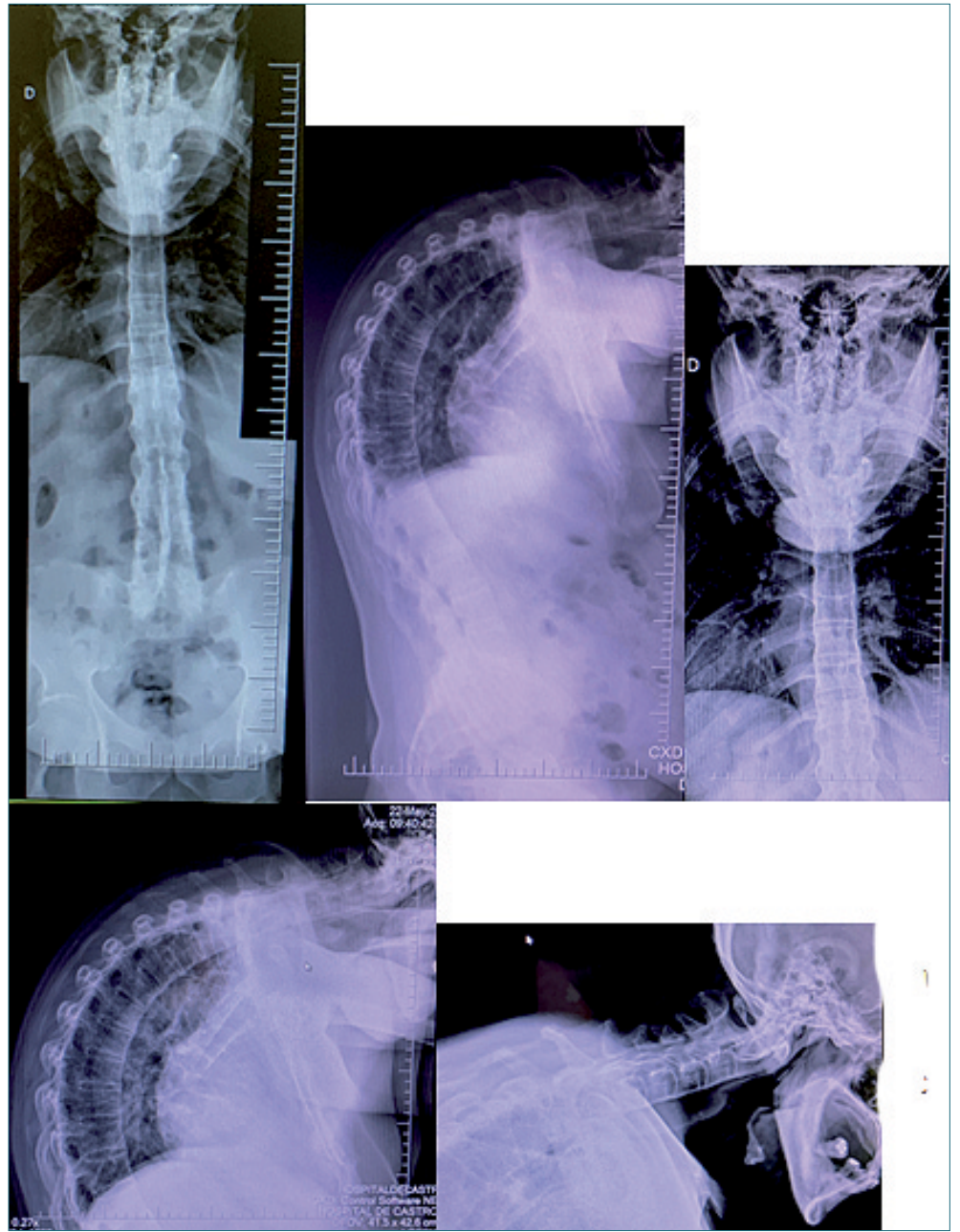

Figura 4. Serie de radiografías de columna que muestra las alteraciones típicas de la EA con una imagen en "caña de bambu".
Como ya se mencionó, la dificultad en el manejo de la vía aérea es una de las mayores causas de morbimortalidad anestésica[11],[12] y tanto en las Guías de manejo de la vía aérea difícil de la ASA 2013 como en el "algoritmo de manejo vía aérea difícil" de la SACHILE en el 2012 propone como alternativa al uso del fibrobroncoscopio en el enfrentamiento del paciente con predictores de mayor complejidad de VAD la evaluación vigil con videolaringoscopio[2],[3].

El videolaringoscopio ha demostrado en varios estudios que es un instrumento efectivo para la intubación traqueal. Proporciona una mejor visión de la laringe y permite una intubación exitosa. H.Y. Lai et al., en el 2006, realiza un trabajo con este "nuevo" dispositivo en 20 pacientes que padecen EA y predictores de VAD. Compara el grado de Cormack y score de porcentaje de apertura de la glotis con laringoscopio
Macintosh \#3 y videolaringoscopio (glidescope), ya que usa el videolaringoscopio para pacientes que rechazan la intubación vigil con fibrobroncoscopio realizando una intubación nasal bajo anestesia general, en este trabajo se concluye que el glidescope provee una mejor visión laringoscópica que la del larongoscopio directo. La mayoría de los pacientes con EA que presentaban Cormack y Lehane III y IV por laringoscopía directa pueden ser exitosamente intubados por un glidescope. Su uso para intubación traqueal puede ser una opción alternativa en estos pacientes que prefieren su manejo de vía aérea bajo anestesia[13]. No se debe olvidar que para la intubación con fibra óptica se recomienda la anestesia del nervio glosofaríngeo más anestésico local, lo que suma una destreza más a la de la manipulación del fibrobroncoscopio.

Se han escrito 2 estudios[14],[15] con un gran nú- 
mero de pacientes que afirman que el VL consigue intubar el $94 \%$ y al $99 \%$ respectivamente de los pacientes en los que la LD no lo logra gracias a la mejora de la visión laríngea.

Kilicaslan et al., en el contexto de VAD no predicha nuevamente comparó la visión de la laringe del videolaringoscopio (C-MAC) y la laringoscopía directa con laringoscopio Macintosh, concluyendo que el videolaringoscopio es eficiente y seguro como dispositivo de recate primario en intubaciones no exitosas inesperadas[16].

En una revisión sistemática y metaanálisis realizado el 2017 por B.M.A. Pieters et al., que en base a 9 estudios que sumaron 1.329 pacientes en los que se comparó el uso de videolaringoscopio en anestesistas experimentados se demostró que éste, en compara- ción con la laringoscopía enfrentando a una VAD anticipada, resultaba mejor visión, menor trauma de mucosa y mejoraba la tasa de éxito al primer intento[17].

En conclusión, actualmente el videolaringoscopio es una alternativa válida al fibrobroncoscopio para la intubación de pacientes con VAD anticipada, candidatos de intubación vigil, y que en comparación este último tendría como ventajas, que es más accesibles a los anestesistas, para los anestesistas con experiencia la destreza en el manejo del videolaringoscopio no presenta mayores complicaciones y suele ser muy fácil mantener la expertise dado que suele ser un devise que está siempre disponible, y no requiere una desinfección especial para su limpieza, los cuidados del videolaringoscopio son más simples que los del fibrobroncoscopio.

\section{Referencias}

1. Langeron $\mathrm{O}$, Masso $\mathrm{E}$, Huraux C, Guggiari M, Bianchi A, Coriat $P$, et al. Prediction of Difficult Mask Ventilation. Anesthesiology [Internet]. Ovid Technologies (Wolters Kluwer Health); 2000 May;92(5):1229-36. http:// dx.doi.org/10.1097/00000542200005000-00009

2. Apfelbaum JL, Hagberg CA, Caplan RA, Blitt CD, Connis RT, Nickinovich DG, et al.; American Society of Anesthesiologists Task Force on Management of the Difficult Airway. Practice guidelines for management of the difficult airway: an updated report by the American Society of Anesthesiologists Task Force on Management of the Difficult Airway. Anesthesiology. 2013 Feb;118(2):25170. https://doi.org/10.1097/ ALN.0b013e31827773b2 PMID:23364566

3. Recomendaciones de la Sociedad de Anestesiología de Chile para el Manejo de la Vía Aérea Difícil. Rev Chil Anest. 2012;41:166-78.

4. Recomendaciones de la Sociedad de Anestesiología de Chile para el Manejo de la Vía Aérea Difícil. Rev Chil Anest,. 2012; 41: 166178.

5. Sieper J, Braun J, Rudwaleit M, Boonen A, Zink A. Ankylosing spondylitis: an overview. Ann Rheum Dis. 2002 Dec;61 Suppl 3:iii8-18. https://doi. org/10.1136/ard.61.suppl_3.iii8 PMID:12381506

6. Wittmann FW, Ring PA. Anaesthesia for hip replacement in ankylosing spondylitis. J R Soc Med. 1986 Aug;79(8):457-9. https:// doi.177/014107688607900808 PMID:3761289

7. Rajesh K, Lawrence H. Spondyloarthropaties. Am Fam Phys. 2004;69:2854-60.

8. Sieper J, Braun J, Rudwaleit M, Boonen A, Zink A. Ankylosing spondylitis: an overview. Ann Rheum Dis. 2002 Dec;61 Suppl 3:iii8-18. https://doi. org/10.1136/ard.61.suppl_3.iii8 PMID:12381506

9. Rajesh K, Lawrence H. Spondyloarthropaties. Am Fam Phys. 2004;69:2854-60.

10. Baeza C, Fornet I, Garcés G. Anestesia para la intervención de osteoclasia vertebral en un paciente con espondilitis anquilopoyética grave de predominio cervical. Rev Esp Anestesiol Reanim. 1993 Nov-Dec;40(6):365-7. PMID:8134679

11. Woodward LJ, Kam PC. Ankylosing spondylitis: recent developments and anaesthetic implications. Anaesthesia. 2009 May;64(5):540-8. https:// doi.org/10.1111/j.13652044.2008.05794.x PMID:19413825

12. Prediction of difficult mask ventilation. Anesthesiology. 2000;92(5):1217-8. PMID:10781262

13. Caplan RA, Posner KL, Ward RJ, Cheney FW. Adverse respiratory events in anesthesia: a closed claims analysis. Anesthesiology. 1990 May;72(5):828-33. https:// doi.org/10.1097/00000542199005000-00010 PMID:2339799

14. Lai HY, Chen IH, Chen A, Hwang FY, Lee $Y$. The use of the GlideScope for tracheal intubation in patients with ankylosing spondylitis. Br J Anaesth. 2006 Sep;97(3):419-22. https:// doi.org/10.1093/bja/ael133 PMID:16829671 
15. Asai T, Liu EH, Matsumoto $S$, Hirabayashi Y, Seo N, Suzuki A, et al. Use of the Pentax-AWS in 293 patients with difficult airways. Anesthesiology. 2009 Apr;110(4):898-904. https://doi.org/10.1097/ ALN.0b013e31819c45e5 PMID:19293707

16. Aziz MF, Healy D, Kheterpal S, Fu RF, Dillman D, Brambrink AM. Routine clinical practice effectiveness of the Glidescope in difficult airway management: an analysis of 2,004 Glidescope intubations, complications, and failures from two institutions. Anesthesiology. 2011 Jan; 114(1):3441. https://doi.org/10.1097/ ALN.0b013e3182023eb7 PMID:21150569

17. Alper Kilicaslan, Ahmet Topal, Aybars Tavlan, Atilla Eroly Seref Otelcioglu. Eficacia delvideolaringoscopioC-MAC $®$ en elmanejo de intubacionesnoexitosas. Rev
Bras Anestesiol. 2014;64(1):625.

18. Pieters BM, Maas EH, Knape JT, van Zundert AA. Videolaryngoscopy vs. direct laryngoscopy use by experienced anaesthetists in patients with known difficult airways: a systematic review and metaanalysis. Anaesthesia. 2017 Dec;72(12):1532-41. https:// doi.org/10.1111/anae. 14057 PMID:28940354 